\title{
Maltrato entre escolares: Diagnóstico en estudiantes de nivel secundaria en la Ciudad de México
}

\author{
Minerva Ramírez Almaraz *, Frida Díaz Barriga Arceo y Rafael López Flores \\ Universidad Nacional Autónoma de México, México
}

(Recibido 16 Mayo, 2015; Aceptado 27 Junio, 2015)

\begin{abstract}
RESUMEN: La finalidad del artículo fue indagar el tipo de maltrato presente entre adolescentes mexicanos. Participaron 480 estudiantes de segundo grado de secundaria (13.72 años de edad promedio con una desviación estándar de .60), pertenecientes a 12 escuelas públicas del Distrito Federal, México. Se adaptó el cuestionario del Defensor del Pueblo de España (2007) para la recopilación de los datos. Los resultados obtenidos indican que en todas las escuelas participantes se manifestaron los diferentes tipos de maltrato contemplados en el instrumento, incluidos los que ocurren mediante el uso de las TIC. El porcentaje de estudiantes espectadores (que habían observado maltrato) fue mayor respecto a quienes manifestaron haber recibido y realizado alguno de los tipos de maltrato escolar investigados. En cuanto a las diferencias entre hombres y mujeres, ellas manifestaron realizar más frecuentemente maltrato indirecto, mientras que los varones indicaron realizar con mayor frecuencia tanto conductas de acoso directo como indirecto.
\end{abstract}

Palabras clave: maltrato entre escolares, educación secundaria, encuesta de auto reporte, diferencias de género, ciberbullying.

\section{Diagnosis of type of Bullying in eight grade students from Mexico City}

ABSTRACT: The purpose of this paper was to investigate the type of bullying among Mexican adolescents. 480 students (average 13.72 years with a standard deviation of .60) attended eight grade in 12 public schools in Mexico City responded an adaptation of the questionnaire of the Ombudsman of Spain (2007). Data from the survey indicated that in all participating schools exist the different types of abuse explored by the instrument, including those that occur through the use of ICT. The percentage of spectators (students who had observed abuse) was higher compared to those who said they had received and performed some of the types of investigated school bullying. As for the differences between gender, women expressed more frequently perform indirect abuse, whereas males reported conduct most often both direct and indirect harassment.

Keywords: bullying, middle school, survey research, genre differences, cyberbullying.

\section{INTRODUCCIÓN}

El término de maltrato entre escolares (bullying) hace referencia a un fenómeno escolar que ha sido estudiado desde la década de los setenta. No es 
una situación nueva, pero se ha malinterpretado el uso de la palabra bullying. Incidentes aislados y no malintencionados suelen decir que se refieren a este fenómeno. Olweus, entre 1986 y 1991, definió el acoso e intimidación de la siguiente manera: "Un alumno es agredido o se convierte en víctima cuando está expuesto, de forma repetida y durante un tiempo, a acciones negativas que lleva a cabo otro alumno o varios de ellos" (Olweus 2006, p.25). Al respecto Harris y Petrie (2006) definen el acoso en la escuela como: "Una conducta intencionadamente agresiva y dañina de una persona o un grupo de personas con mayor poder, dirigida repetidamente contra una persona con menos poder, normalmente sin que medie la provocación" (Harris y Petrie, 2006, p. 18). En ambas definiciones encontramos dos constantes, por un lado, las conductas que dañan a la víctima y por otro, que una persona o varias son quienes realizan este tipo de malos tratos.

Aunque en las definiciones anteriores, los autores mencionan que deben ser considerados aquellos maltratos que se realizan de manera repetida, es relevante, tomar en cuenta situaciones particulares, que pueden resultar significativas y dolorosas para el alumnado y no necesariamente debe existir la característica de ser repetida en el tiempo. Las agresiones pueden ser realizadas por una persona o por un grupo de personas, quienes dirigen sus agresiones hacia un individuo o hacia varios (Olweus 2006; Harris y Petrie 2006).

Un elemento relevante que se hace presente en el maltrato es una relación asimétrica de poder. Olweus (2006) precisa que esta característica puede ser imaginaria o real; y es en la atribución que realiza la víctima, la que hace que se sienta vulnerable e indefensa de las agresiones que vive. Fernández (2007) resalta que el abuso se realizará por parte de quien sea más fuerte, o bien, de quienes se muestren más fuertes al exhibir su dominio hacia sus pares. Lo anterior es complementario con lo planteado por Ortega (2007), quien explica la relación del modelo de dominio- sumisión, que se refiere a la actuación de un alumno/a que se autopercibe con mayor poder o con un poder incontrolado, en comparación con el resto del grupo escolar, debido a que los demás no se atreven a decirle que no los moleste. Cuando este dominio ocurre en repetidas ocasiones sin que se ponga un alto a esa conducta, es entonces cuando "descubre que los demás son capaces de aguantar sus impertinencias y eso hace que las siga realizando" (Ortega, 2007, p. 25).

En cuanto a las consecuencias que se han investigado sobre los/as agresores/as y víctimas encontramos las investigaciones de Olweus (2006), las cuales mostraron la relación entre ser agresor o agresora y presentar problemas conductuales tales como delincuencia o alcoholismo. Y también resalta que al llegar a la edad adulta, manifiestan tener mayor grado de depresión que aquellos que no acosaron a otros estudiantes, existe mayor probabilidad de abandonar los estudios. Sobre el grupo de estudiantes que padecen maltrato en la escuela, las consecuencias pueden ser no querer asistir a ésta porque sienten miedo y el malestar emocional que provoca en ellos ser víctimas, puede estimular baja en su rendimiento académico.

Debido a todo lo mencionado, se ha generado interés por realizar investiga- 
ciones en diferentes países y todas concuerdan en que el maltrato es un fenómeno que se ha extendido entre la población escolar, por lo que se estima que uno de cada seis estudiantes recibe algún tipo de maltrato por parte de sus pares, y que es durante los 9 y 14 años cuando se presenta con mayor frecuencia, es decir, entre los últimos años de primaria y los primeros de secundaria (El Defensor del Pueblo, 2007). Otra autora comenta sobre su frecuencia: "es mayor en la adolescencia temprana (13-15 años), en los cursos de educación secundaria obligatoria, en los que se experimenta una mayor dependencia del grupo de compañeros, en aquellos que suelen resultar más difíciles para el profesorado de secundaria" (Díaz-Aguado, 2005, s/n página).

Una de las manifestaciones de maltrato en las escuelas es el denominado ciberbullying, que es definido por Del Rey, Flores, Garmendia, Martínez, Ortega y Tijerina (2011), como aquel que se lleva a cabo por medio de las TIC, al ser ejercido, sufrido y observado desde cualquier lugar y en cualquier momento, es decir, desde medios online o por medio del teléfono celular. Es una experiencia ambigua que trastoca diferentes ámbitos de la vida. Para este grupo de autores, el ciberbulllying es considerado como un bullying indirecto, que es posible realizarse a través del hostigamiento, la exclusión y la manipulación (Del Rey et al, 2011).

Sobre el estado del conocimiento del bullying encontramos las siguientes aportaciones que nos sirven para contextualizar la temática de estudio. Garaigordobil, Martínez y Aliri (2013), en un estudio que realizaron con 178 adolescentes de ambos sexos con altas puntuaciones en victimización tuvieron significativamente menor nivel de autoestima. Realizaron un análisis de regresión en donde dos variables resultaron predictoras de victimización: baja autoestima y menor nivel de edad. Cabeza (2010) encontró diferencias entre hombres y mujeres, con un rango de edad de 9 a 14 años, en la manera en que en mayor medida maltratan a sus pares. Los varones utilizan más la fuerza física y las mujeres acosan a sus iguales de forma solapada. Raimundo y Seixas (2009) en una investigación encontraron que los varones manifiestan realizar más conductas de acoso directo, mientras que las mujeres indican protagonizar maltratos de forma indirecta. Castillo y Pacheco (2008) realizaron un estudio en donde se ponen de manifiesto diferencias cuantitativas entre el bullying manifestado por estudiantes españoles y mexicanos. En México, se acentúan más los resultados de maltrato entre pares. Las autoras resaltan que estas diferencias no son motivo de sorpresa, debido a que en España han promovido acciones para mejorar la convivencia y la prevención de violencia entre iguales. En México aún no existen programas establecidos con impacto. Señalan otro dato interesante, debido a que las formas de maltrato con una mayor incidencia corresponden al abuso verbal. Las autoras acotan que parece que en la Estado de Yucatán, México, existe la costumbre de percibir de manera natural los chismes, los insultos o los apodos ofensivos, sin cuestionar que se trata de armas severas contra la autoestima y la integridad moral de las personas.

Oliveros, Amemiya, Condorimay, Oliveros, Barrientos y Rivas (2012) en- 
contraron que en estudiantes de los últimos años de primaria y durante la secundaria, la presencia de ciberbullying resultó muy alta, por lo que debe ser considerado un problema de salud pública. En otro estudio, Mc Guckin, Pauline, Cummins y Christopher (2010) reportan resultados sobre situaciones de bullying en el sistema educativo de Irlanda del Norte. Examinaron la prevalencia tanto de manera presencial, como a través del cyberbullying. Los malos tratos entre pares fueron frecuentes en toda la muestra. Las experiencias personales de implicación en los problemas del tema investigado se asociaron a peores puntuaciones en bienestar psicológico.

En México concretamente, que es el país donde se desarrolló la presente investigación, resulta indispensable visualizar que existe maltrato entre escolares dentro de las escuelas, lo que nos ayudará a buscar un abordaje pertinente que dé respuesta al derecho que tiene el alumnado de asistir a una escuela donde se promueva un entorno benéfico libre de violencia. Mirar el problema desde una óptica cercana nos apoyará para saber hacia dónde caminar en estas situaciones que de ninguna manera tenemos que ignorar. Algunas estadísticas recopiladas en México, por el Instituto Nacional de Evaluación Educativa [INEE] en el 2007, señalan que alumnos de tercer grado de secundaria manifestaron: 13.2\% han sufrido amenazas por parte de otro alumno, 14\% ha recibido golpes de sus mismos compañeros dentro de la escuela. De acuerdo con un artículo de Gómez (2008), el Secretario de Educación del D.F., Axel Didriksson dio a conocer los resultados de una Encuesta sobre violencia en las escuelas del Distrito Federal, en la que se indica que siete de cada diez alumnos han sido agresores, víctimas o testigos. Los cuestionarios aplicados en este contexto no cuentan con las características metodológicas que tiene el Defensor del Pueblo de España (2007), por este motivo se eligió adaptar dicho instrumento para adolescentes mexicanos, lo cual se detalla en la parte correspondiente del instrumento. En el 2014, el cuestionario adaptado en la presente investigación fue utilizado por Figueroa (2014), para la realización de una investigación sobre el tema de violencia entre compañeros de educación secundaria.

Los objetivos del presente estudio son: 1. Adaptar el cuestionario del Defensor del Pueblo de España (2007), a la población de educación secundaria del Distrito Federal, México; 2. Detectar la incidencia y magnitud del maltrato entre escolares y el ciberbullying, en estudiantes de segundo grado de escuelas secundarias de la Ciudad de México; 3. Determinar si se presentan variaciones porcentuales en la incidencia en las manifestaciones de maltrato en el rol de agresor/a entre estudiantes del sexo masculino y femenino.

\section{METODOLOGÍA}

\section{Muestra}

Durante el ciclo escolar 2008-2009, se aplicó el cuestionario a 480 estudiantes de segundo grado de secundaria, de la Ciudad de México, de los cuales el 50\% 
pertenecían al turno matutino y $50 \%$ al turno vespertino; en cuanto al género el $50 \%$ eran hombres y $50 \%$ mujeres. Las edades de los participantes fluctuaron entre los 13 y 16 años, la media estadística fue de 13.7 años con una desviación típica de .60. En el presente estudio se manejó el muestreo aleatorio simple, por medio del cual se eligieron a 12 escuelas.

\section{Instrumento}

Se utilizó el Cuestionario del Defensor del Pueblo de España (2007), debido a que este considera cuestiones metodológicas relevantes como indagar acciones concretas y no el concepto global. Se incluyen preguntas de forma directa, en el presente estudio sólo se rescatan los reactivos referentes a la incidencia del maltrato para quienes se reconocen como víctimas (14 reactivos), agresores (14 reactivos) y espectadores (14 reactivos); asimismo, los referentes a la incidencia del ciberbullying para quienes manifiestan los roles de víctimas (8 reactivos), agresores ( 8 reactivos) y espectadores ( 8 reactivos). En el Defensor del Pueblo de España 2007, se agregan estas preguntas sobre maltrato a través de las TIC, de manera abierta en este estudio las incluimos con el formato de las otras preguntas. El cuestionario sigue la misma organización del Defensor del Pueblo de España 2007. Incluye una introducción donde se define el maltrato entre escolares e indicaciones sobre el procedimiento para su contestación. La incidencia de maltrato y de cibercullying se evaluó en una escala tipo Likert ordinal, con niveles de respuesta: nunca, a veces, frecuentemente y siempre. Este cuestionario investiga directamente sobre cada tipo de maltrato en particular, de tal manera que puede establecerse la incidencia de cada uno de ellos y sus características específicas con mayor precisión.

\section{Tipo de estudio}

El diseño de la investigación consiste en un estudio descriptivo tipo encuesta.

\section{Procedimiento}

Los cuestionarios fueron aplicados en las instalaciones de cada una de las escuelas en el turno correspondiente. En cada uno de los 12 colegios participaron 20 alumnos y 20 alumnas de segundo grado, quienes fueron seleccionados al azar por el equipo directivo de las escuelas. Una vez reunido el grupo participante, se les explicaba el objetivo de la investigación y se hacía hincapié en que este estudio tendría un carácter anónimo. La aplicación del instrumento tuvo una duración media de 50 minutos. 


\section{RESULTADOS}

Iniciaremos este apartado presentando los resultados de acuerdo a los objetivos planteados para este estudio. En primer lugar, la adaptación del cuestionario del Defensor del Pueblo de España (2007) a la población de educación secundaria del Distrito Federal, México. Dicho procedimiento consistió en los siguientes pasos:

1. Reunión con docentes españoles para encontrar aquellas palabras que eran desconocidas para estudiantes mexicanos y en donde buscamos el referente que correspondiera. Si bien está en idioma castellano, los modismos propios de España son ajenos al contexto mexicano.

2. En una evaluación por jueces se les solicitó que hicieran una revisión a la versión preliminar, los expertos fueron académicos universitarios especialistas en desarrollo humano, bullying y metodología de la investigación. Cada uno de los jueces hizo las puntualizaciones que consideró necesarias.

3. Se piloteó el cuestionario con 40 alumnos de primero, segundo y tercer grado. En este punto los alumnos manifestaron que les parecía muy extenso y que había preguntas que se repetían, con base en lo cual se hicieron una serie de modificaciones.

4. Para conocer la consistencia interna de los reactivos se aplicó el alfa de Cronbach obteniéndose un valor de .91, lo cual indica que tiene una consistencia muy elevada.

En las preguntas de los cuestionarios referentes a la incidencia se utilizaron cuatro categorías de respuesta para estimar la intensidad de la conducta: "nunca", "a veces", "a menudo" y "siempre". No obstante, para hacer más clara la presentación de evidencias, las hemos agrupado solamente en los puntajes totales, es decir, presentamos la ocurrencia total de respuestas para cada tipo de maltrato. Se citarán, únicamente, las tres conductas que ocupen los porcentajes más elevados en las preguntas como víctimas, agresores/as y espectadores/as, tanto en el maltrato entre escolares, como en el ciberbullying.

Estimación de la incidencia de cada tipo de maltrato a partir de las respuestas de las víctimas, agresores/as y espectadores/as

En respuesta al segundo objetivo, referente a la detección de la incidencia y magnitud del maltrato entre escolares en estudiantes de segundo grado de escuelas secundarias de la Ciudad de México, exponemos a continuación los datos obtenidos por el alumnado participante cuando responden a las preguntas acerca de que si han sido objeto de distintos tipos de maltrato, es decir, cuando se sitúan en la perspectiva de víctimas de maltrato por parte de otros. 
Valoración de las respuestas de las víctimas

En la tabla 1, se presentan los porcentajes obtenidos para cada uno de los maltratos indagados en este estudio. El primer lugar lo ocupa me ponen apodos ofensivos, con un $52.30 \%$, en segundo lugar se situó la conducta hablan mal de mí con un $51.30 \%$ y con $50 \%$ tenemos la conducta de me insultan.

Estimación del número de agresores y agresoras para cada tipo de maltrato

En este apartado se muestra la incidencia, partiendo de la información proporcionada por quienes reconocen ser agresores. Como se puede observar en la tabla 1, se presenta la incidencia de quienes reconocieron agredir a sus pares. $\mathrm{El}$ orden de mayor incidencia de los distintos tipos de maltrato fue: insulto con $61.1 \%$, seguida de ignoro $54.50 \%$ y pongo apodos ofensivos con $57.9 \%$.

La percepción de ocurrencia de maltrato según los testigos

Como podemos observar en la tabla 1 , se obtuvieron altos porcentajes del alumnado que declara ser testigo/a de las distintas modalidades de maltrato, en donde se comprueba que es mayor el número de espectadores/as de maltrato entre escolares en relación con las víctimas y agresores/as. Los resultados obtenidos con mayor porcentaje fueron: poner apodos ofensivos con $94.85 \%$, seguida de hablar mal de alguien con $94.55 \%$ y esconder cosas con $92.95 \%$. 
Tabla 1. Porcentaje Total de Estudiantes que Declaran Haber Sido Víctimas, Agresores/as, y Espectadores/as de Diferentes Tipos de Maltrato

\begin{tabular}{|c|c|c|c|c|}
\hline & Tipos de maltrato & Víctimas & Agresores/as & Espectadores/as \\
\hline \multirow[t]{3}{*}{ Exclusión social } & Ignorar & $28.55 \%$ & $54.50 \%$ & $86.70 \%$ \\
\hline & No dejar participar & $21.50 \%$ & $25.4 \%$ & $77.75 \%$ \\
\hline & Insultar & $50 \%$ & $61.1 \%$ & $84.15 \%$ \\
\hline \multirow[t]{4}{*}{ Agresión verbal } & Poner apodos ofensivos & $52.30 \%$ & $57.9 \%$ & $94.85 \%$ \\
\hline & Hablar mal de alguien & $51.30 \%$ & $46.10 \%$ & $94.55 \%$ \\
\hline & Difundir rumores negativos & $37.75 \%$ & $25.85 \%$ & $85.45 \%$ \\
\hline & Esconder cosas & $29.60 \%$ & $26.25 \%$ & $92.95 \%$ \\
\hline \multirow{2}{*}{$\begin{array}{l}\text { Agresión } \\
\text { indirecta }\end{array}$} & Romper cosas & $9.95 \%$ & $11.35 \%$ & $72.70 \%$ \\
\hline & Robar cosas & $26.25 \%$ & $6 \%$ & $71.45 \%$ \\
\hline $\begin{array}{l}\text { Agresión física } \\
\text { directa }\end{array}$ & Pegar & $14.40 \%$ & $33.15 \%$ & $82.90 \%$ \\
\hline Amenazas/ & $\begin{array}{l}\text { Amenazar para que sienta } \\
\text { miedo }\end{array}$ & $15.40 \%$ & $19.1 \%$ & $79.25 \%$ \\
\hline \multirow[t]{2}{*}{ Chantajes } & Obligar con amenazas & $4.60 \%$ & $9.85 \%$ & $51.5 \%$ \\
\hline & Amenazar con armas & $1.40 \%$ & $3.40 \%$ & $22.10 \%$ \\
\hline Acoso sexual & Acosar sexualmente & $8.75 \%$ & $4.35 \%$ & $30.50 \%$ \\
\hline
\end{tabular}

Como se observa en la figura 1, el porcentaje de estudiantes que señalan ser testigos/as de diferentes tipos de maltrato es mayor con relación a víctimas y agresores/as. En esta se puede comparar el comportamiento de los protagonistas del maltrato entre escolares, en relación a las conductas del maltrato que se han incluido en este estudio. 


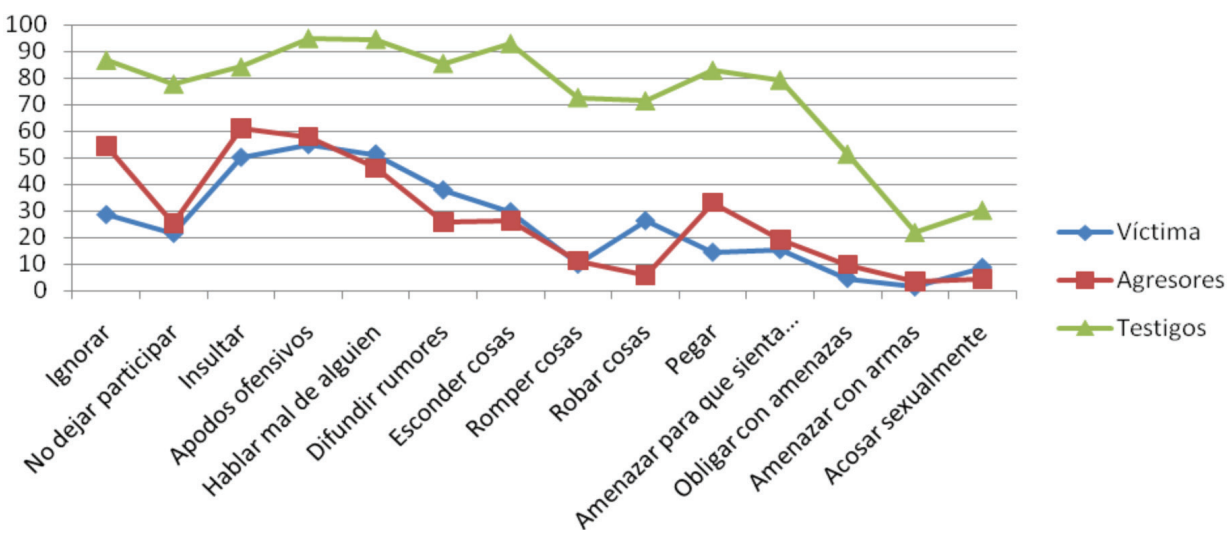

Figura 1.Comparación del porcentaje de víctimas, agresores/as y testigos/as en los diferentes tipos de maltatro.

\section{Incidencia de maltrato a través de las TIC}

En lo referente a las víctimas a través de las TIC, como se observa en la tabla 2 , encontramos que las tres conductas con más alto índice de porcentaje son: $h a-$ blan mal de mí con un $26.70 \%$, en segundo lugar encontramos difunden rumores negativos sobre mí con un $20.90 \%$, me ponen apodos con un $18.35 \%$. En cuanto a los/as agresores/as a través de las TIC, como se observa en la tabla 2, hallamos que las tres conductas con más alto porcentaje fueron, en primer lugar hablo mal de alguien $22.70 \%$, seguida por pongo apodos $20.25 \%$ y en tercer lugar insulto con $19.20 \%$. En lo referente a los/as espectadores/ras de maltrato por medio de las TIC, encontramos que en la tabla 2, las tres conductas con más alto porcentaje fueron, en primer lugar hablo mal de alguien $72.35 \%$, seguida de difunden rumores negativos con $69.85 \%$ y poner apodos que ridiculizan con $67.5 \%$. 
Tabla 2. Porcentaje de Estudiantes que Declara Ser Victima, Agresores/as y Espectadores/as de Distintas Situaciones de Ciberbullying

\begin{tabular}{|c|c|c|c|c|}
\hline & Tipos de maltrato & Víctimas & Agresores/as & Espectadores/as \\
\hline \multirow{5}{*}{ Agresión verbal } & Insultar & $16.85 \%$ & $19.20 \%$ & $63.35 \%$ \\
\hline & Poner apodos ofensivos & $18.35 \%$ & $20.25 \%$ & $67.50 \%$ \\
\hline & Hablar mal de alguien & $26.70 \%$ & $22.70 \%$ & $72.35 \%$ \\
\hline & Difundir rumores negativos & $20.90 \%$ & $14.45 \%$ & $69.85 \%$ \\
\hline & Amenazar para que sienta miedo & $8.70 \%$ & $7.75 \%$ & $49.65 \%$ \\
\hline Amenazas/ & Subir videos sin su permiso & $3.75 \%$ & $5.85 \%$ & $60.60 \%$ \\
\hline Chantajes & Subir fotografías sin su permiso & $10.40 \%$ & $7.10 \%$ & $54.85 \%$ \\
\hline Acoso sexual & Acosar sexualmente & $11.25 \%$ & $2.75 \%$ & $27.55 \%$ \\
\hline
\end{tabular}

Variaciones porcentuales en la incidencia de agresoras y agresores

El tercer objetivo, se propuso para determinar si se presentaban diferencias porcentuales en las manifestaciones de maltrato entre escolares de segundo grado en las conductas que utilizan para agredir entre el sexo masculino y el femenino.

Considerando los resultados obtenidos, en la tabla 3, se ponen de manifiesto mayor porcentaje que el alumnado participante exteriorizó las agresiones que realizan hacia otros compañeros/as. Se puede observar que las mujeres manifestaron mayor porcentaje de maltrato en tres conductas, las cuales son parte del acoso indirecto; mientras que los varones alcanzaron porcentajes más elevados en 11 conductas, lo que indica que los varones manifestaron realizar, con mayor frecuencia, tanto conductas de acoso directo como indirecto. 
Tabla 3. Porcentaje de Agresores/as en los Distintos Tipos de Maltrato Desglosado por Género

\begin{tabular}{lcc}
\hline Tipos de maltrato & Alumnas & Alumnos \\
\hline Ignoro & $28.55 \%$ & $26.05 \%$ \\
\hline No dejo participar & $9.20 \%$ & $16.25 \%$ \\
\hline Insulto & $26.30 \%$ & $34.75 \%$ \\
\hline Pongo apodos que ofenden & $23.15 \%$ & $34.80 \%$ \\
\hline Hablo mal de alguien & $26.45 \%$ & $19.6 \%$ \\
\hline Difundo rumores negativos & $14.60 \%$ & $11.25 \%$ \\
\hline Escondo cosas & $7.10 \%$ & $19.15 \%$ \\
\hline Rompo cosas & $3.35 \%$ & $7.90 \%$ \\
\hline Robo cosas & $1.25 \%$ & $4.80 \%$ \\
\hline Pego & $10.85 \%$ & $22.25 \%$ \\
\hline Amenazo para que sienta miedo & $6.70 \%$ & $12.90 \%$ \\
\hline Obligo con amenazas & $2.90 \%$ & $6.90 \%$ \\
\hline Amenazo con armas & $1.05 \%$ & $2.50 \%$ \\
\hline Acoso sexualmente & & $3.55 \%$ \\
\hline
\end{tabular}

\section{CONCLUSIONES}

Iniciaremos este apartado presentando los resultados de acuerdo a los objetivos planteados. En primer lugar, la adaptación del cuestionario del Defensor del Pueblo de España (2007), a la población de educación secundaria del Distrito Federal, México. Para conocer la consistencia interna de los reactivos de aplicó el alfa de Cronbach y el resultado de .91 , nos permite confiar en los resultados del presente estudio.

Sobre el segundo objetivo sobre la incidencia del maltrato entre escolares y el ciberbullying, a partir de los datos descritos en el apartado anterior, podemos afirmar que todos los tipos de maltrato por los que se indagó en este estudio se hacen presentes en las 12 escuelas investigadas. De acuerdo con la incidencia estimada por el conjunto de estudiantes, en los porcentajes globales, que manifiestan las víctimas de las diferentes modalidades de maltrato, el porcentaje más 
alto corresponde a las agresiones verbales, en sus diferentes manifestaciones. De acuerdo a lo encontrado por Garaigordobil et al. (2013), altas puntuaciones en victimización presentan menor nivel de autoestima. Debemos hacer algo para prevenir la "cicatrices mentales" que tienen como consecuencias el ser víctima de bullying (Olweus, 2006). De acuerdo a la información que nos dan quienes se reconocen como agresores, en los porcentajes globales, encontramos que los maltratos más frecuentes son las agresiones verbales y en segundo lugar tenemos a la exclusión social. Desde la perspectiva de espectadores, el porcentaje de maltrato reportado es mayor respecto a quienes declaran haber recibido o ejercido los diferentes tipos de maltrato incluidos en esta investigación. Siendo las conductas con mayor porcentaje las referentes a la agresión verbal agresión física indirecta y la exclusión social.

En cuanto al ciberbullying encontramos que todos los tipos de maltrato por los que se indagaron se hicieron presentes en las 12 escuelas participantes. De acuerdo con Oliveros et al. (2012), la presencia de este tipo de maltrato a través de las TIC, nos deben llevar a considerar este tipo de conductas como un problema de salud pública. Reflexionando sobre estos datos debemos tomar en cuenta lo encontrado por Mc Guckin, et al. (2010), en donde la implicación en situaciones de bullying y ciberbullying se asocia a puntuaciones bajas en bienestar psicológico. Como educadores no queremos satanizar el uso de las TIC, el cual sería un grave error. Utilizadas de manera estratégica, ética y segura, las TIC nos brindan la posibilidad de acceder a información que ayuda, educa y permite la convivencia, no sólo a los educandos si no al mundo entero. ¿Qué es lo que proponemos los autores? Enseñar a utilizar las TIC de una manera responsable y estratégica, favorecer el conocimiento de las consecuencias psicológicas y legales que resultan de conductas que se realizan en el ciberbullying, en aras de promover la ciudadanía digital.

En respuesta al tercer objetivo sobre la incidencia de quienes agreden, de acuerdo al género concuerda con lo que Olweus (2006); Harris y Petrie (2006), Raimundo y Seixas (2009) y Cabeza (2010), quienes señalan acerca de que las mujeres son más propensas al acoso indirecto en comparación con los varones. Un dato relevante que debemos resaltar es que los varones reconocieron realizar mayor número de conductas de agresión que las mujeres; ellas por su parte solamente en 3 conductas tuvieron mayor frecuencia, mientras que el porcentaje de conductas de maltrato que ellos realizan es mayor en 11 conductas de las 14 por las que se investiga en este estudio. Las consecuencias negativas no sólo las tienen las víctimas, sino que también los agresores, tal como lo cita Olweus (2006) y Harris y Petrie (2006), ya que estos últimos tienen el riesgo de tener más adelante problemas conductuales como delincuencia o alcoholismo, mayor grado de depresión en comparación con quienes no acosaron a otros compañeros, entre otras repercusiones negativas que se pueden rescatar de diversos estudios sobre el tema.

En México, se hace evidente la importancia de sensibilizar y concientizar a las autoridades educativas sobre la problemática que viven los y las estudiantes 
de educación secundaria, ya que este es un tema que no acaba de abordarse de manera clara y con elementos sólidos para ser afrontado como es requerido por quienes manifiestan que padecen de malos tratos por parte de sus compañeros de escuela. Por ello, debemos optar por la prevención, en donde enseñemos a la comunidad escolar a rechazar estas conductas que dañan la integridad de conjunto de estudiantes que son víctimas de sus pares. Esto se vincula con los resultados de Castillo y Pacheco (2008), que nos marcan un foco de atención al respecto: en estudiantes mexicanos se acentuó el maltrato entre pares, en comparación con estudiantes españoles. En México no se han establecido programas adecuados de largo alcance e impacto real de afrontamiento para el bullying.

Este estudio fue realizado durante el ciclo escolar 2008- 2009, lo cual es una limitación, por el tiempo que ha transcurrido desde que se concluyó. Pero el aporte de la validación del instrumento ha servido para otras investigaciones, como es el caso de Figueroa (2014).

\section{AGRADECIMIENTOS}

A la Dra. Cristina del Barrio Martínez, catedrática de la Universidad Autónoma de Madrid, por todo su apoyo teórico y metodológico en la realización del presente estudio.

Al Dr. Cruz Pérez Pérez, profesor de la Universidad de Valencia, por su apoyo en la adaptación del cuestionario.

A la Dra. Sandra Espino Datsira, profesora de la Universidad de Barcelona, por su colaboración en la revisión de este documento.

Al Lic. Marcos Verdejo, académico de la UNAM, por su asesoría en la estadística.

A la DGAPA de la UNAM a través del proyecto PAPIIT IN304114-3.

\section{REFERENCIAS}

Aguilera, M., Muñoz, G. y Orozco, A. (2007). Disciplina, violencia y consumo de sustancias nocivas a la salud en escuelas primarias y secundarias de México. México: INEE.

Cabeza, H. (2010). ¿Qué ocurre en el aula costarricense? Los niños y las niñas que maltratan a sus compañeros. Actualidades Investigativas en Educación, 10(3), 1- 21. Extraído el 15 de Mayo de 2015 desde http://www.redalyc.org/ articulo.oa? $\mathrm{id}=44717980011$

Castillo, C., y Pacheco M. (2008). Perfil del maltrato (bullying) entre estudiantes de secundaria en la ciudad de Mérida, Yucatán. Revista Mexicana de Investigación Educativa, 13 (38), 825- 842. Extraído el 15 de Mayo de 2015 desde http://www.comie.org.mx/documentos/rmie/v13/n038/pdf/v13n038art006es. pdf

Defensor del pueblo (2007). Violencia escolar: el maltrato entre iguales en la educación secundaria obligatoria 1999-2006 (Nuevo estudio y actualización del Informe 2000, Informes, estudios y documentos). Madrid: Defensor del Pueblo, España. 
Del Rey, R., Flores, J., Garmendia, M. Martínez, G., Ortega, R. y Tejerina, O. (2011). Protocolo de actuación escolar ante el ciberbullying. Gobierno Vasco Eusko Jaurlaritza.

Díaz Aguado M. (2005). Por qué se produce la violencia escolar y cómo prevenirla. Revista Iberoamericana de Educación, 37. Extraído el 15 de Mayo de 2015 desde http://www.rieoei.org/rie37a01.htm

Fernández, I. (2007). Prevención de la violencia y resolución de conflictos: El clima escolar como factor de calidad. Madrid: Narcea.

Figueroa, M. (2014). Violencia entre compañeros de secundaria en un pueblo del Distrito Federal. Tesis de Doctorado en Pedagogía, Facultad de Filosofía y Letras, Universidad Nacional Autónoma de México.

Garaigordobil, M., Martínez, V. y Aliri. J. (2013). Autoestima, empatía y conducta agresiva en adolescentes víctimas de bullying presencial. European Journal of Investigation in Health, Psychology and Education, 3 (1), 29- 40. Extraído el 15 de Mayo de 2015 desde http://www.siis.net/documentos/ficha/199144.pdf

Gómez, L. (2008). La violencia en las escuelas, fomentada por los medios y la familia: Didriksson. La Jornada. Extraído el 10 de enero de 2009 desde http://www.jornada.unam.mx/2008/12/04/index.php?section=capital\&article $=043$ n 1 cap

Harris, S. y Petrie, G. (2006). El acoso en la escuela. Los agresores, las víctimas y los espectadores. Barcelona: Paidós.

Mc Guckin, C, Cummins, P. y Lewis, Ch. (2010). f2f and cyberbullying among children in Northern Ireland: Data from the Kids Life and Times Surveys. Psychology, Society, \& Education, 2, (2), 83- 96. Extraído el 15 de Mayo de 2015 desde http://dialnet.unirioja.es/servlet/articulo? codigo $=3444309$

Olweus, D. (2006). Conductas de acoso y amenaza entre escolares. Madrid: Morata.

Olweus, D. (2006). Una revisión general. En Serrano, A. (2006). Acoso y violencia en la escuela. Como detectar, prevenir y resolver el bullying. Barcelona: Ariel.

Oliveros, M.., Amemiya, I., Condorimay, Y., Oliveros, R., Barrientos, A. y Rivas, B. (2012). Ciberbullying - Nueva tecnología electrónica al servicio del acoso escolar en alumnos de dos distritos de Lima, Perú Ciberbullying. Anales de la Facultad de Medicina, 73(1), 13-18.

Ortega, R. (2007). Violencia, agresión y disciplina. En Fernández, I. (2007). Prevención de la violencia y resolución de conflictos. El clima escolar como factor de calidad. Madrid: Narcea.

Raimundo, R. y Seixas, S. (2009). Comportamentos de Bullying no $1^{\mathrm{o}}$ ciclo: estudo de caso numa escola de Lisboa. Interacções. 13, 164-186.

Ramírez, M. (2011). Maltrato entre escolares: Diagnóstico en alumnos de segundo de secundaria de la Delegación Gustavo A. Madero. Tesis de Maestría en Pedagogía, Facultad de Filosofía y Letras, Universidad Nacional Autónoma de México, Distrito Federal, México. 